\title{
HARMONIC FUNCTIONS AND THE STRUCTURE OF COMPLETE MANIFOLDS
}

\author{
PETER LI \& LUEN-FAI TAM \\ Dedicated to Professor Shiing-Shen Chern on his 79th birthday
}

\section{Introduction}

This paper is motivated by previous work of the authors [18] and its application to the study of the structure of complete Kähler manifolds in a subsequent work of the first author [16]. Roughly speaking the main theorem in [18] relates the infinity geometric structure of a certain class of manifolds to the theory of harmonic functions. Let us recall the precise setting.

Let $M$ be a complete noncompact manifold without boundary. Suppose the sectional curvature $K_{M}$ of $M$ is nonnegative outside some compact subset of $M$. Without loss of generality, we may assume that the compact subset is contained in a geodesic ball of radius 1 . By using the argument of Cheeger-Gromoll [5], one concludes rather easily that $M$ has finite topological type. In particular, $M$ has finitely many ends and each end is homeomorphic to the product of a compact manifold with the halfline. In fact, Abresch [1] (also see [2]) proved that a slightly more general assumption on the sectional curvature, which he referred to as asymptotically nonnegatively curved, is sufficient to imply that $M$ has finite topological type. Moreover, the number of ends of $M$ can be estimated by a quantity computable by the pointwise lower bound of the sectional curvature.

In the case when $M$ has nonnegative sectional curvature on $M \backslash B(1)$, the ends of $M$ are given by the connected components of $M \backslash B(1)$. The notions of large and small ends were defined by the authors [18] depending on the volume growth of the end. With these definitions, we may assume the ends of $M$ are given by $s$ small ends $\left\{e_{1}, \cdots, e_{s}\right\}$ and $l$ large ends $\left\{E_{1}, \cdots, E_{l}\right\}$, for some $0 \leq s<\infty$ and $0 \leq l<\infty$ with $s+l \geq 1$. The main result of [18] was to show that the numbers $s$ and $l$ have

Received October 5, 1990. The first author's research was partially supported by a National Science Foundation grant and a John Simon Guggenheim Fellowship. 
function theoretic meaning. To elaborate on this, let us first introduce various spaces of harmonic functions defined on $M$.

Definition 0.1. The space $\mathscr{H}^{\infty}(M)$ is the linear space of all bounded harmonic functions defined on $M$.

Definition 0.2. The space $\mathscr{H}^{+}(M)$ is the linear space spanned by the set of all positive harmonic functions on $M$.

The theorem of [18] asserts that if $M \backslash B(1)$ has nonnegative sectional curvature, and if $l=0$, then $\operatorname{dim} \mathscr{H}^{+}=\operatorname{dim} \mathscr{H}^{\infty}=1$. However, if $l \geq 1$, then $\operatorname{dim} \mathscr{H}^{\infty}=l$ and $\operatorname{dim} \mathscr{H}^{+}(M)=s+l$.

By constructing a metric at infinity (see [13]) of the asymptotically nonnegatively curved manifolds, Kasue later [14] generalized this theorem to that situation. A complete manifold is asymptotically nonnegatively curved means that the sectional curvature $K_{M}$ of $M$ satisfies

$$
K_{M}(x) \geq-k(r(x))
$$

for some nonnegative monotonically nonincreasing continuous function of the distance $r$ to a fixed point, with the property that

$$
\int_{1}^{\infty} t k(t) d t<\infty
$$

These theorems give rise to a natural question. Can the assumption on the sectional curvature be weakened to an assumption on the Ricci curvature instead? Other than just a technical improvement, there are two main practical reasons for this question. Let us first recall Yau's theorem [31] which asserts that a complete manifold with nonnegative Ricci curvature does not admit a nonconstant positive harmonic function. If we, for a moment, ignore the fact that the assumption is on the Ricci curvature instead, one could consider the theorem of Li-Tam as a generalization of Yau's theorem. This can be seen by applying the splitting theorem of Cheeger-Gromoll [4], which asserts that $M$ either has one end or it has two small ends. Hence, if Li-Tam's theorem is valid for nonnegative Ricci curvature, then Yau's theorem would be a corollary. However, it is unfortunate that the argument in [18] and [14] used the infinity structure rather heavily, which in general is not true for manifolds with nonnegative Ricci curvature outside a compact set. Retrospectively, Yau's theorem is rather amazing now that we know of examples by Sha-Yang [27], [28] of positively Ricci curved manifolds with infinite topological type. In any event, Yau's theorem and the subsequent work of Li-Yau [22] on the heat equation indicate that the analysis is still not so complicated even though the topology could be. This philosophy is encouraged by the work of Donnelly 
and Cheng. Donnelly [10] first observed that the space of bounded harmonic functions on a manifold with nonnegative Ricci curvature outside a compact set is finite dimensional. Cheng [8] showed that the space of positive harmonic functions is of finite dimension on the same class of manifolds. In the same paper, he also gave an estimate on the dimension of $\mathscr{H}^{\infty}(M)$ by a constant depending only on the dimension on $M$, the diameter of the set where $M$ has negative Ricci curvature, and the lower bound of the Ricci curvature on $M$.

Another motivation for relaxing the curvature assumption on Li-Tam's theorem is given by the work of the first author [16]. Using the harmonic functions constructed in [18], he studied the structure of complete Kähler manifolds with nonnegative sectional curvature outside a compact set. For the purpose of possible applications in algebraic geometry, it is quite desirable to generalize this structural theorem or even a part of this theorem to curvature, in particular, the Ricci curvature or the holomorphic bisectional curvature.

The purpose of this paper is to show that for most practical purposes, a more general version of Li-Tam's theorem is valid with an inequality. In particular, this answers some of the questions posted above. Throughout this paper, the Riemannian manifold $M$ is assumed to be complete, noncompact, and without boundary. Let us first consider the following definitions.

Definition 0.3. A manifold is said to be parabolic if it does not admit a positive Green's function. Conversely, a nonparabolic manifold is one which admits a positive Green's function.

Definition 0.4. An end $E$ of a manifold $M$ is an unbounded component of the complement of some compact subset $D$ of $M$. In this case, we say that $E$ is an end corresponding to $D$.

It is also easy to see [19] that parabolicity of a manifold depends only on its infinity behavior. Hence, it makes sense to speak of whether an end is parabolic or not.

Definition 0.5. An end $E$ is said to be parabolic if it is the only end of some complete parabolic manifold without boundary. This is the same as saying that $E$ could be extended to a complete parabolic manifold by attaching a compact set to its boundary. Conversely, an end $E$ is nonparabolic if it is nonparabolic after extending to a complete manifold without boundary by attaching a compact set to its boundary.

It is clear that if $D_{1} \subset D_{2}$ are compact subsets of $M$, then the number of ends corresponding to $D_{1}$ is less than or equal to the number of ends corresponding to $D_{2}$. Hence we say that $M$ has finitely many ends if 
there exists $b<\infty$ such that the number of ends corresponding to $D$ is less than or equal to $b$ for any compact subset $D \subset M$. One also observes that in this case there exist an integer $b_{0}<\infty$ and a compact subset $D_{0} \subset M$ such that the number of ends corresponding to $D$ is $b_{0}$ for all compact subsets $D$ containing $D_{0}$. We say that $b_{0}$ is the number of ends of $M$. Moreover, it is known that if $M$ has a nonparabolic end, then $M$ is itself nonparabolic.

In the case when $M$ satisfies the hypothesis of Li-Tam's theorem or Kasue's theorem, it was shown that an end is large if and only if it is nonparabolic. It is also known [29] (also see [22]) that if a complete manifold has nonnegative Ricci curvature everywhere, then the notion of a large end is also equivalent to that of a nonparabolic end. However, due to technical reasons, it is still not known for manifolds with nonnegative Ricci curvature outside a cocompact set whether the notion of large end and nonparabolic end are equivalent. On the other hand, it was shown by the authors [20] that if an end is nonparabolic then it must be a large end. We shall also point out that Varopoulos [30] has an example of a large end which is parabolic.

Let us now define the following spaces of harmonic functions on a complete manifold.

Definition 0.6. The space $\mathscr{H}_{D}^{\infty}(M)$ is the space of bounded harmonic functions on $M$ which has finite Dirichlet integral.

Definition 0.7. The space $\mathscr{H}^{0}(M)$ is the linear space spanned by the set of harmonic functions on $M$ which are bounded on one side at each end. More precisely, $\mathscr{H}^{0}(M)$ is spanned by those harmonic functions $f$ which have the property that there is a compact subset $D \subset M$, such that $f$ is bounded from above or below on each of the ends corresponding to D.

Clearly, we have the monotonic relations

$$
\text { \{constants }\} \subset \mathscr{H}_{D}^{\infty}(M) \subset \mathscr{H}^{\infty}(M) \subset \mathscr{H}^{+}(M) \subset \mathscr{H}^{0}(M) .
$$

The key result of this paper (Theorem 2.1) is to construct a subspace $\mathscr{H}^{\prime}(M)$ of $\mathscr{H}^{0}(M)$ such that the number of ends of $M$ is bounded by $\operatorname{dim} \mathscr{C}^{\prime}(M)$. When the manifold is nonparabolic, this upper bound can be sharpened so that the number of ends is bounded from above by $\operatorname{dim} \mathscr{H}^{+}(M)$, and the number of nonparabolic ends is bounded from above by $\operatorname{dim} \mathscr{H}_{D}^{\infty}(M)$. In both cases, we construct subspaces of $\mathscr{H}^{+}(M)$ and $\mathscr{H}_{D}^{\infty}(M)$, respectively, whose dimension gives precisely the number of ends being considered. The surprising part about this theorem is that there are no curvature assumptions imposed on $M$ at all. 
We would like to remark that the notion of Martin boundary is directly related to the boundary of the space $\mathscr{H}^{+}(M)$. However, if the manifold is parabolic, then one can conclude that $\mathscr{H}^{+}(M)=\{$ constant $\}$. Hence, in view of Theorem 2.1, perhaps one should consider the boundary which is related to the space $\mathscr{H}^{0}(M)$ instead. Another interesting point is that Grigor' yan [12] has shown that the condition $\operatorname{dim} \mathscr{H}_{D}^{\infty}(M)>1$ is a quasiisometric invariant. It is also true that the notion of parabolicity is a quasiisometric invariant. On the other hand, an example of Lyons [25] showed that the condition $\operatorname{dim} \mathscr{H}^{+}(M)>1$ is, in general, not a quasi-isometric invariant. This leads us to speculate that there should be a subspace of $\mathscr{H}^{+}(M)$ which is quasi-isometrically stable for the purpose of detacting the number of ends.

In the last two sections of this paper we will give some applications of Theorem 2.1 to Riemannian geometry ( $\$ 3$ ) and Kähler geometry ( $(4)$. In Theorem 3.1 we show that if the Ricci curvature of $M^{n}$ satisfies

$$
\operatorname{Ric}_{M}(x) \geq-k(r(x))
$$

for some monotonically nonincreasing continuous function $k(r)$ of the distance $r(x)=d(p, x)$ to a fixed point $p \in M$ such that

$$
\int_{0}^{\infty} r^{n-1} k(r) d r<\infty,
$$

then there exists a constant $C(n, k)>0$ depending on $n$ and the function $k$ alone so that $\operatorname{dim} \mathscr{H}^{\prime}(M) \leq C(n, k)$. In particular, the number of ends of $M$ is not greater than $C(n, k)$. In the special case when the Ricci curvature is nonnegative on $M \backslash B(1)$, the argument can be simplified substantially and one can estimate the dimension of the larger space $\mathscr{H}^{0}(M)$ in terms of a constant depending on $n$ and the lower bound of the Ricci curvature on $B(1)$. Of course, by Theorem 2.1, this implies that the number of ends has the same upper bound.

We would like to point out that Liu has independently proven a ballcovering lemma for manifolds with nonnegative Ricci curvature on $M \backslash B(1)$ and with the additional assumption that the sectional curvature is bounded from below on $M$. As a consequence, the number of ends can be estimated in terms of the dimension and the lower bound of the sectional curvature. At the same time, Cai [3] has also proved an estimate on the number of ends (similar to ours) without the assumption on the sectional curvature. After reading Cai's preprint, Liu [24] has been able to modify his argument so that the assumption on the lower bound of the sectional curvature is deleted. In the work of Liu and Cai the methods 
of proof are purely Riemannian geometric and are completely different in approach in comparison to ours.

In $\S 4$, we assume that $M$ is a complete Kähler manifold. Then by using an argument of [16] and a vanishing theorem of [23], we will show in Theorem 4.2 that if $M^{m}$ has Ricci curvature bounded from below by

$$
\operatorname{Ric}_{M}(x) \geq-\delta(m) r^{-2}
$$

for a sufficiently small $\delta(m)>0$ depending only on $m$, and if $M$ is nonparabolic, then $M$ must have only one end. By imposing a much weaker curvature assumption in terms of some $L^{q}$ integral on the Kähler manifold $M$, we will also show (Theorem 4.1) that $M$ can only have one nonparabolic end. This integral curvature assumption was first considered by Yau and the first author in their work [23] where they proved a generalization of Huber's theorem in higher dimensions.

\section{Green's function and parabolicity}

In this section we will recall some of the fact about green's functions on complete Riemannian manifolds. The authors considered the question of whether any complete manifold admits a symmetric Green's function. It turned out that the answer is affirmative and its existence was first proved by Malgrange [26]. A construction procedure was recently developed by the authors [19], and as a consequence these Green's functions satisfy some nice properties which are useful for the purpose of constructing harmonic functions in $\S 2$. Let us first outline our construction procedure.

Let $\Omega_{i}$ be a monotonic increasing sequence of compact subdomains of $M$, which gives an exhaustion of the manifold. Consider the symmetric Green's function $G_{i}(x, y)$ with Dirichlet boundary condition on $\Omega_{i}$. For a fixed point $p \in M$ and a compact subset $D$ of bounded as functions in the $y$-variable. It was also shown (see Remark 1 of [19]) that the existence of a geodesic ball $B_{p}(R)$ and a corresponding end which admits a positive harmonic function with its infimum achieved at infinity is equivalent to the condition that $M$ admits a positive Green's function. In particular, the end which supports the positive harmonic function is a nonparabolic end. Moreover, if $f$ is a nonconstant harmonic function mentioned above defined on a nonparabolic end $e$ with $-\infty<\inf _{e} f=\min _{\partial e} f$, then by the maximum principle by taking $e_{0}$ to be $e \backslash\{$ an open neighborhood of $\partial e\}$, $f$ will satisfy $-\infty<\inf _{e_{0}} f<\min _{\partial e_{0}} f$. Hence such an $f$ does not exist. This proves the following proposition. 
Proposition 1.1. $E$ is a nonparabolic end of $M$ if and only if there exists a nonconstant harmonic function $f$ defined on $E$ with the property that $-\infty<\inf _{E} f<\min _{\partial E} f$.

In fact, by using $f$ as a barrier, one could prescribe boundary condition on $\partial E$.

Lemma 1.1. If $E$ is a nonparabolic end, then there exists a harmonic function $\phi$ defined on $E$ with the following properties:

(1) $1 \geq \phi \geq 0$ on $E$;

(2) $\phi=1$ on $\partial E$;

(3) $\phi\left(p_{i}\right) \rightarrow 0$ for some sequence of points in $E$ such that $p_{i} \rightarrow \infty$;

(4) $\phi$ has finite Dirichlet integral over $E$.

Proof. Let $E$ be a nonparabolic end corresponding to $B_{p}(R)$. For $r>R$, let us consider the harmonic function $\phi_{r}$ on $E \cap B_{p}(r)$ with the boundary conditions that $\phi_{r}=1$ on $\partial E$ and $\phi_{r}=0$ on $E \cap \partial B_{p}(r)$. By Proposition 1.1 there exists a harmonic function on $E$ such that $-\infty<$ $\inf _{E} f<\min _{\partial E} f$. By translation, we may assume that $\inf _{E} f=0$ and that there exists a sequence of points $p_{i} \in E$ with $p_{i} \rightarrow \infty$ such that

$$
f\left(p_{i}\right) \rightarrow 0 \text {. }
$$

By the maximum principle and the boundary conditions, clearly

$$
0 \leq \phi_{r} \leq f / \min _{\partial E} f
$$

on $E \cap B_{p}(r)$. Hence, the gradient estimate implies that the family of functions $\phi_{r}$ converges uniformly to a harmonic function $\phi$ on compact subsets of $E$ satisfying

$$
0 \leq \phi \leq f / \min _{\partial E} f \text { on } E
$$

and

$$
\phi=1 \text { on } \partial E \text {. }
$$

Clearly, by (1.1), $\phi$ satisfies condition (3). The fact that $\phi$ has finite Dirichlet integral follows from the argument of Theorem 1.1 in [16] or Lemma 1.4 below. q.e.d.

In the case when $M$ does not admit one of the barriers mentioned above, it was shown that for any fixed compact subset $D$ of $M \backslash\{p\}$, by passing through a subsequence the sequence of functions

$$
g_{i}(y)=G_{i}(p, y)-\min _{x \in D} G_{i}(p, x)
$$

converges to a Green's function on a compact subset. Observe that since the oscillations of $G_{i}(p, y)$ are uniformly bounded on $D$, the sequence 
of functions

$$
h_{i}(y)=G_{i}(p, y)-\max _{x \in D} G_{i}(p, x)
$$

also has a convergent subsequence which converges to $h(y)$ defined on $M \backslash\{p\}$. However, $h_{i}$ is defined so that $h_{i} \leq 0$ on $\Omega_{i} \backslash D$, hence $h$ is a nonpositive harmonic function on $M \backslash D$. In fact, $\inf _{M \backslash D} h=-\infty$, otherwise $M$ would have a positive Green's function. This construction implies the following lemma.

Lemma 1.2. Let $e$ be the only parabolic end corresponding to a compact subset $B_{p}(R)$ of $M$. Then there exists a harmonic function $\psi$ defined on $e$ with the following properties:

(1) $\psi \geq 0$ on $e$;

(2) $\psi=0$ on $\partial e$; and

(3) $\psi\left(q_{j}\right) \rightarrow \infty$ for some sequence of points in $e$ such that $q_{j} \rightarrow \infty$.

Proof. From the construction described, the Green's function is obtained by taking the limit of the sequence (1.2). In particular, if we take $\Omega_{i}=B_{p}\left(R_{i}\right)$ for a sequence of $R_{i} \rightarrow \infty$, then there exists a sequence of positive harmonic functions $f_{i}$ defined on $e$ given by

$$
f_{i}(y)=\max _{x \in\left(B_{p}(R) \backslash B_{p}(R / 2)\right)} G_{i}(p, x)-G_{i}(p, y)
$$

such that $f_{i} \rightarrow f$. In particular, $f_{i}$ has boundary condition $f_{i}=C_{i}$ for

$$
C_{i}=\max _{x \in\left(B_{p}(R) \backslash B_{p}(R / 2)\right)} G_{i}(p, x) .
$$

Let us now consider the harmonic function $\psi_{i}$ on $e \cap B_{p}\left(R_{i}\right)$ with boundary conditions $\psi=0$ on $\partial e$ and $\psi=C_{i}$ on $e \cap \partial B_{p}\left(R_{i}\right)$. Clearly, by the maximum principle, we have

$$
f_{i}(y)-\max _{x \in \partial e} f_{i}(x) \leq \psi(y) \leq f_{i}(y)
$$

for all $y \in e \cap B_{p}\left(R_{i}\right)$. Since $f_{i} \rightarrow f$, we conclude that there is a positive harmonic function $\psi$ defined on $e$ such that $\psi_{i} \rightarrow \psi$. The function $\psi$ clearly satisfies the desired properties. q.e.d.

We will also give some estimates on the growth of the Dirichlet integral of the barriers constructed in Lemmas 1.1 and 1.2, which will be used in the later sections.

Lemma 1.3. Let $M$ be a complete noncompact manifold and let $f$ be a harmonic function on $M \backslash B_{p}(1)$, where $p$ is a fixed point. Suppose there are $C_{i}>0, R_{i} \rightarrow \infty$, and harmonic functions $f_{i}$ on $B_{p}\left(R_{i}\right) \backslash B_{p}(1)$ with 
$\left.f_{i}\right|_{\partial B_{p}(1)}=0$ and $\left.f_{i}\right|_{\partial B_{p}\left(R_{i}\right)}=C_{i}$ such that $f=\lim _{i \rightarrow \infty} f_{i}$. Then, given any $a>0$,

$$
\int_{M \backslash B_{p}(1)}|\nabla \log (f+a)|^{2} \leq \frac{1}{a} \int_{\partial B_{p}(1)} \frac{\partial f}{\partial r}
$$

and

$$
\int_{B_{p}(R) \backslash B_{p}(1)}|\nabla f|^{2} \leq 4 \sup _{\partial B_{p}(R)} f \times \int_{\partial B_{p}(1)} \frac{\partial f}{\partial r} .
$$

Proof. For simplicity, let us denote $B(r)=B_{p}(r)$. If $R_{i}>R>1$, then

$$
\begin{aligned}
& \int_{B(R) \backslash B(1)}\left|\nabla \log \left(f_{i}+a\right)\right|^{2} \leq \int_{B\left(R_{i}\right) \backslash B(1)}\left|\nabla \log \left(f_{i}+a\right)\right|^{2} \\
& =-\int_{B\left(R_{i}\right) \backslash B(1)} \Delta \log \left(f_{i}+a\right)=-\frac{1}{C_{i}+a} \int_{\partial B\left(R_{i}\right)} \frac{\partial f_{i}}{\partial r}+\frac{1}{a} \int_{\partial B(1)} \frac{\partial f_{i}}{\partial r} \\
& \leq \frac{1}{a} \int_{\partial B(1)} \frac{\partial f_{i}}{\partial r},
\end{aligned}
$$

where we have used the facts that $f_{i}>0, \partial f_{i} / \partial r>0$ on $\partial B\left(R_{i}\right)$, and $f_{i}=0$ on $B(1)$. By letting $i \rightarrow \infty$, and then $R \rightarrow \infty$, inequality (1.3) follows. By observing that (1.3) implies

$$
\int_{B(R) \backslash B(1)}|\nabla f|^{2} \leq \frac{\left(\sup _{B(R) \backslash B(1)} f+a\right)^{2}}{a} \times \int_{\partial B(1)} \frac{\partial f}{\partial r},
$$

and by setting $a=\sup _{\partial B(R)} f=\sup _{B(R) \backslash B(1)} f$, we obtain inequality (1.4).

Lemma 1.4. Let $M$ be a complete noncompact manifold and $f$ a harmonic function on $M \backslash B_{p}(1)$, where $p$ is a fixed point. Suppose there exist $R_{i} \rightarrow \infty$ and harmonic functions $f_{i}$ on $B_{p}\left(R_{i}\right) \backslash B_{p}(1)$ with $\left.f_{i}\right|_{\partial B_{p}(1)}=$ $\left.f\right|_{\partial B_{p}(1)}$ and $\left.f_{i}\right|_{\partial B_{p}\left(R_{i}\right)}=0$ such that $f=\lim _{i \rightarrow \infty} f_{i}$. Then

$$
\int_{M \backslash B_{p}(1)}|\nabla f|^{2} \leq \int_{\partial B_{p}(1)} f \frac{\partial f}{\partial r} .
$$

Proof. Since $f_{i}$ is harmonic on $B_{p}\left(R_{i}\right) \backslash B_{p}(1)$ and $f_{i}=0$ on $\partial B_{p}\left(R_{i}\right)$, we have

$$
\int_{B_{p}\left(R_{i}\right) \backslash B_{p}(1)}\left|\nabla f_{i}\right|^{2}=\int_{\partial B_{p}(1)} f_{i} \frac{\partial f_{i}}{\partial r} .
$$

The estimate follows by letting $i \rightarrow \infty$.

\section{Existence of harmonic functions}

Let us now consider the set of ends corresponding to the compact set $B_{p}(R)$. Suppose $\left\{e_{1}, \cdots, e_{s(R)}\right\}$ are the set of parabolic ends for some 
$0 \leq s(R)<\infty$, and $\left\{E_{1}, \cdots, E_{l(R)}\right\}$ are the set of nonparabolic ends for some $0 \leq l(R)<\infty$ with $s(R)+l(R) \geq 1$. Clearly the counting functions $s(R)$ and $l(R)$ are monotonically nondecreasing functions of $R$. If $M$ has finitely many ends, then $s(R) \rightarrow s$ and $l(R) \rightarrow l$ for some integers $s<\infty$ and $l<\infty$. We will now construct harmonic functions on $M$, which are adopted to the parabolicities of the ends.

Theorem 2.1. Let $M$ be a complete manifold. If $\operatorname{dim} \mathscr{H}^{0}(M)<\infty$, then $M$ must have finitely many ends. In particular, if $s$ and $l$ denote the number of parabolic ends and the number of nonparabolic ends, respectively, then

$$
s+l \leq \operatorname{dim} \mathscr{H}^{0}(M)
$$

Moreover, if $l \geq 1$, then

$$
s+l \leq \operatorname{dim} \mathscr{H}^{+}(M)
$$

and

$$
l \leq \operatorname{dim} \mathscr{H}_{D}^{\infty}(M)
$$

Proof. To prove the theorem it suffices to show that $s(R)$ and $l(R)$ satisfy the estimate stated in the theorem for all values of $R$. Let $R>0$ be arbitrary. For simplicity, let us denote $s(R)=s$ and $l(R)=l$. Let us consider the ends corresponding to $B_{p}(R)$ as denoted above. For each nonparabolic end $E_{i}$ there is a harmonic function $\phi_{i}$ defined on $E_{i}$ and a sequence of points $\left\{p_{k}^{i}\right\}_{k=1}^{\infty} \in E_{i}$ satisfying the properties of Lemma 1.1. Also, for each parabolic end $e_{j}$ there is a harmonic function $\psi_{j}$ defined on $e_{j}$ and a sequence of points $\left\{q_{k}^{j}\right\}_{k=1}^{\infty} \in e_{j}$ satisfying the properties of Lemma 1.2.

To prove (2.3), let us first assume that $l \geq 2$. In this case, for any nonparabolic end, say $E_{i}$, by an argument of the authors [18, Theorem 6.1 ] one can construct a bounded harmonic function $f_{i}$ on $M$ with the following properties:

(1) $f_{i}\left(p_{k}^{i}\right) \rightarrow 1$ as $p_{k}^{i} \rightarrow \infty$;

(2) $f_{i}\left(p_{k}^{j}\right) \rightarrow 0$ as $p_{k}^{j} \rightarrow \infty$ for and $j \neq i$; and

(3) $0<f_{i}<1$.

By the construction and Lemma 1.4, the functions $f_{i}$ have finite Dirichlet integrals. Moreover, $\left\{f_{i}\right\}_{i=1}^{l}$ forms a linearly independent set. In fact, if we have the identity

$$
0=\sum_{j=1}^{l} a_{j} f_{j}
$$


for a set of constants $\left\{a_{1}, \cdots, a_{l}\right\}$, then by evaluating on the sequence of points $p_{k}^{i}$ and letting $k \rightarrow \infty$, we have

$$
0=\sum_{j=1}^{l} a_{j} \lim _{k \rightarrow \infty} f_{j}\left(p_{k}^{i}\right)=a_{i} .
$$

Hence, $\operatorname{dim} \mathscr{H}_{D}^{\infty}(M) \geq l$. Since the constant functions are in $\mathscr{H}_{D}^{\infty}(M)$, the estimate is automatic when $l=1$.

To prove (2.2), we will construct positive harmonic functions which distinguish the parabolic ends. Let $e_{i}$ be a fixed parabolic end. Let us consider the harmonic function $h_{i, r}$ defined on $B_{p}(r)$ such that $h_{i, r}=0$ on $\partial B_{p}(r) \backslash e_{i}$ and $h_{i, r}=\psi_{i}$ on $e_{i} \cap \partial B_{p}(r)$. We claim that $h_{i, r}$ is bounded uniformly on $\partial e_{i}$ for all $r$. To see this, let us denote $\alpha(r)=\max _{\partial e_{i}} h_{i, r}$. Clearly $\alpha(r)>0$ by the maximum principle. If $\alpha(r) \rightarrow \infty$, then let us consider the function

$$
H_{r}=h_{i, r} / \alpha(r) .
$$

This nonnegative harmonic function satisfies $\max _{\partial e_{i}} H_{r}=1$. By passing through a subsequence, the gradient estimate implies that $H_{r} \rightarrow H$, which is a nonnegative harmonic function on $M$ with the following properties:

(1) $\max _{\partial e_{i}} H=1$;

(2) $H \leq 1$ on $M \backslash e_{i}$; and

(3) $H\left(p_{k}^{j}\right) \rightarrow 0$ for all $0 \leq j \leq l$.

The last property follows from the fact that $H_{r} \leq \phi_{j}$ on $E_{j}$. Moreover, since $H_{r} \leq 1 \leq \psi_{i} / \alpha(r)+1$ on $\partial e_{i} \cup\left(e_{i} \cap \partial B_{p}(r)\right)$, the maximum principle yields

$$
H_{r} \leq \psi_{i} / \alpha(r)+1 \text { on } e_{i} \cap B_{p}(r) .
$$

Hence by taking the limit as $r \rightarrow \infty$ and using the fact that $\alpha(r) \rightarrow \infty$, we have $H \leq 1$ on $e_{i}$. Together with properties (1) and (2) of $H$, the strong maximum principle gives that $H \equiv 1$ on $M$. However, this is a contradiction to property (3). Therefore, we conclude that $\alpha(r)$ is bounded from above also. Let us denote the upper bound by $\alpha$. The functions $\psi_{i}+\alpha$ and $\psi_{i}$ can now be used as upper and lower barriers to show that the sequence of functions $h_{i, r}$ must converge to some harmonic function $h_{i}$ defined on $M$ which has the following properties:

(1) $h_{i}\left(q_{k}^{i}\right) \rightarrow \infty$;

(2) $h_{i}\left(p_{k}^{j}\right) \rightarrow 0$ for all $1 \leq j \leq l$; and

(3) $0 \leq h_{i} \leq \alpha$ on $e_{j}$ for all $j \neq i$ and $1 \leq j \leq s$. 
In fact, the difference $\tau_{r}=h_{i, r}-\psi_{i}$ is a harmonic function defined on $e_{i} \cap B_{p}(r)$ with boundary conditions $\tau_{r}=h_{i, r}>0$ on $\partial e_{i}$ and $\tau_{r}=0$ on $e_{i} \cap \partial B_{p}(r)$. Since $h_{i, r} \rightarrow h_{i}$, the function $\tau_{r} \rightarrow h_{i}-\psi_{i}$, which is a bounded harmonic function on $e_{i}$.

To see that the set of functions $\left\{f_{1}, \cdots, f_{l}, h_{1}, \cdots, h_{s}\right\}$ forms a linear independent set, we consider the linear combination

$$
0=\sum_{i=1}^{l} a_{i} f_{i}+\sum_{j=1}^{l} b_{j} h_{j} .
$$

By evaluating the sequence of points $\left\{p_{k}^{i}\right\}$ and letting $k \rightarrow \infty$, we conclude that $a_{i}=0$. Evaluating at the sequence of points $\left\{q_{k}^{j}\right\}$ and letting $k \rightarrow \infty$, we conclude that $b_{j}=0$ by properties (1) and (3) of the $h_{i}$ 's. Hence estimate (2.2) is valid.

To prove (2.1) we may assume that $l=0$. The estimate is obvious if $s=1$, so let us assume that $s \geq 2$. We will construct harmonic functions on $M$ such that it is bounded on one side at each end. For values of $R<r<t<\infty$ and a constant $a \geq 0$, let us define the harmonic function $g_{r t}$ on $B_{p}(t) \backslash\left(e_{1} \backslash B_{p}(r)\right)$ with the following properties:

(1) $g_{r t}=-a \psi_{1}$ on $e_{1} \cap \partial B_{p}(r)$;

(2) $g_{r t}=\psi_{2}$ on $e_{2} \cap \partial B_{p}(t)$; and

(3) $g_{r t}=0$ on $\bigcup_{j=3}^{s}\left(e_{j} \cap \partial B_{p}(t)\right)$.

For a fixed $r$, clearly $\min _{\partial e_{2}} g_{r t} \geq-a \max _{e_{1} \cap \partial B_{p}(r)} \psi_{1}=-A$ for $A \geq 0$. We claim that $M_{t}=\max _{\partial e_{2}} g_{r t}$ is bounded from above for all $t>r$. Suppose there is a sequence $t_{i} \rightarrow \infty$ such that $0<M_{i}=M_{t_{i}} \rightarrow \infty$. Let us define the function $G_{i}=M_{i}^{-1} g_{r t_{i}}$. Clearly, $G_{i}$ satisfies $-A / M_{i} \leq G_{i} \leq 1$ on $\partial e_{2}$ and $0 \leq G_{i}=\psi_{2} / M_{i}$ on $e_{2} \cap \partial B_{p}(t)$. Hence, by the maximum principle,

$$
\frac{-A}{M_{i}} \leq G_{i} \leq 1+\frac{\psi_{2}}{M_{i}} \text { on } e_{2} \cap B_{p}(t) .
$$

Moreover, by the boundary conditions of $G_{i}$, we have $-A / M_{i} \leq G_{i} \leq 1$ on $B_{p}(t) \backslash\left(e_{2} \cup\left(e_{1} \backslash B_{p}(r)\right)\right)$. Therefore, by passing through a subsequence, $G_{i} \rightarrow G$, which is a harmonic function defined on $M \backslash\left(e_{1} \backslash B_{p}(r)\right)$ satisfying $0 \leq G \leq 1$, with $G=0$ on $e_{1} \cap \partial B_{p}(r)$ and $\max _{\partial e_{2}} G=1$. This implies that $G$ is a nonconstant harmonic function defined on $M \backslash\left(e_{1} \backslash B_{p}(r)\right)$ which achieves an interior maximum on $\partial e_{2}$. This is a contradiction. Hence the $M_{t}$ are bounded for all $t$.

Let $t_{i} \rightarrow \infty$ be a sequence such that $M_{i}=M_{t_{i}} \rightarrow B<\infty$. The same argument as above shows that the sequence of functions $g_{r t_{i}} \rightarrow g_{r}$, which 
is a harmonic function on $M \backslash\left(e_{1} \backslash B_{p}(r)\right)$ satisfying

$$
-A+\psi_{2} \leq g_{r} \leq \max _{\partial e_{2}} g_{r}+\psi_{2} \text { on } e_{2},
$$

and has boundary condition $g_{r}=-a \psi_{1}$ on $e_{1} \cap \partial B_{p}(r)$. Also $B=$ $\max _{\partial e_{2}} g_{r}$. In fact, we can say more about $g_{r}$. The harmonic function $g_{r}-\min _{\partial e_{2}} g_{r}-\psi_{2}$ is bounded on $e_{2}$ and is nonnegative on $\partial e_{2}$. Since $e_{2}$ is parabolic, we have

$$
\inf _{e_{2}}\left(g_{r}-\min _{\partial e_{2}} g_{r}-\psi_{2}\right)=\min _{\partial e_{2}}\left(g_{r}-\min _{\partial e_{2}} g_{r}-\psi_{2}\right) \geq 0 \text {. }
$$

Therefore $g_{r}-\min _{\partial e_{2}} g_{r} \geq \psi_{2} \geq 0$ on $e_{2}$.

Let us also analyze the behavior of $g_{r}$ at the other ends $e_{j}$ for $j=$ $3, \cdots, s$. Since $g_{i}=0$ on $e_{j} \cap \partial B_{p}\left(t_{i}\right)$ and $g_{i} \rightarrow g_{r}$, the above argument shows that $g_{r}$ is bounded at $e_{j}$. Again, Proposition 1.1 implies that

$$
\min _{\partial e_{j}} g_{r} \leq g_{r} \leq \max _{\partial e_{j}} g_{r}
$$

on $e_{j}$ for $j=3, \cdots, s$.

We will now consider the set of all $g_{r}$ for $r>R$ and all $a \geq 0$. Since we are now allowing the constant $a \geq 0$ to vary, we denote the harmonic functions constructed on $M \backslash\left(e_{1} \backslash B_{p}(r)\right)$ by $g_{r}^{a}$. Clearly, if we take $a=0$, then $g_{r}^{0}$ is nonnegative. If we define $\min _{B_{p}(2 R)} g_{r}^{a}=m_{r}^{a}$, $\max _{B_{p}(2 R)} g_{r}^{a}=M_{r}^{a}$, and $\omega_{r}^{a}=M_{r}^{a}-m_{r}^{a}$ for $a \geq 0$ and $r>3 R$, then $m_{r}^{0}>0$ for all $r>3 R$. Let us now denote $W=\left\{(a, r) \mid m_{r}^{a} \geq 0\right\} . W$ is nonempty, since it contains $(0, r)$. In particular, $W \cap\{(a, r)\} \neq \varnothing$ for any fixed $r>3 R$. We claim that for $(a, r) \in W$, the oscillations $\omega_{r}^{a}$ of $g_{r}^{a}$ are uniformly bounded on $B_{p}(2 R)$. If there is a sequence of $a_{i}$ and a sequence of $r_{i}$ such that $\omega_{i}=\omega_{r_{i}}^{a_{i}} \rightarrow \infty$, let us consider the functions defined by

$$
F_{i}=\left(g_{i}-m_{i}\right) / \omega_{i},
$$

where $g_{i}=g_{r_{i}}^{a_{i}}$ and $m_{i}=m_{r_{i}}^{a_{i}}$. Then $F_{i}$ have the following properties:

(1) $0=\min _{B_{p}(2 R)} F_{i}$;

(2) $1=\max _{B_{p}(2 R)} F_{i}$;

(3) $0 \leq F_{i} \leq 1$ on $e_{j}$ for all $j=3, \cdots, s$;

(4) $F_{i}=\left(\psi_{2}+\theta_{i}-m_{i}\right) / \omega_{i}$, where $\theta_{i}$ is a harmonic function on $e_{2}$ bounded by $m_{i} \leq \theta_{i} \leq M_{i}$; and

(5) $F_{i} \leq\left(-a_{i} \psi_{1}-m_{i}\right) / \omega_{i}+1 \leq 1$ on $e_{1} \cap B_{p}\left(r_{i}\right)$, because $F_{i} \leq 1$ on $\partial e_{1}$ and $F_{i}=\left(-a_{i} \psi_{1}-m_{i}\right) / \omega_{i} \leq 0$ on $e_{1} \cap \partial B_{p}\left(r_{i}\right)$. 
Hence, by passing through a subsequence and using the fact that $\omega_{i} \rightarrow$ $\infty$, we have $F_{i} \rightarrow F$, which is a harmonic function satisfying:

(1) $0=\min _{B_{p}(2 R)} F$;

(2) $1=\max _{B_{p}(2 R)} F$;

(3) $0 \leq F_{i} \leq 1$ on $e_{j}$ for all $j=3, \cdots, s$;

(4) $F=h$, where $h$ is a harmonic function on $e_{2}$ bounded by $0 \leq$ $h \leq 1 ;$ and

(5) $F \leq 1$.

If the sequence $r_{i} \rightarrow \infty$, then $F$ will be a nonconstant harmonic function on $M$, which is bounded by above by 1 . This is a contradiction to the fact that $M$ has no nonparabolic end. If the sequence $r_{i}$ is bounded, say $\lim \sup _{i} r_{i}=r>3 R$, then $F$ is only defined on $M \backslash\left(e_{1} \backslash B_{p}(r)\right)$ with boundary condition $F \leq 0$ on $e_{1} \cap \partial B_{p}(r)$. Thus by Proposition 1.1 we conclude that $F$ is a nonconstant harmonic function which achieves an interior maximum on $B_{p}(2 R)$. Again, this is a contradiction. Hence the $\omega_{r}^{a}$ are bounded for all $(a, r) \in W$. Let us denote this bound by $\omega$.

We now claim that for each fixed $r>3 R$, we can find a value $a_{r}=a \geq 0$ such that $m_{r}^{a}=0$. By continuity, it suffices to show that for a fixed $r$, $m_{r}^{a} \leq 0$ for some value of $a \geq 0$. If this is not the case, then $m_{r}^{a} \geq 0$ for all $a \geq 0$. Similar to the argument above, as a sequence of functions in $a$, the functions $K^{a}=g_{r}^{a}-m_{r}^{a}$ satisfy:

(1) $0=\min _{B_{p}(2 R)} K^{a}$;

(2) $\omega=\max _{B_{p}(2 R)} K^{a}$;

(3) $0 \leq K^{a} \leq \omega$ on $e_{j}$ for all $j=3, \cdots, s$;

(4) $K^{a}=\psi_{2}+h^{a}-m_{r}^{a}$, where $h^{a}-m_{r}^{a}$ is a bounded harmonic function bounded by 0 and $\omega$; and

(5) $K^{a} \leq-a \psi_{1}-m_{r}^{a}+\omega \leq-a \psi_{1}+\omega$ on $e_{1} \cap B_{p}(r)$.

In particular, $0 \leq K^{a} \leq-a \psi_{1}+\omega$ on $e_{1} \cap B_{p}(2 R)$. This gives a contradiction by taking $a \rightarrow \infty$. In fact, this argument shows that $a \leq$ $\omega / \max _{e_{1} \cap B_{p}(2 R)} \psi_{1}$, which is independent of $r$.

Take a sequence of $r_{i} \rightarrow \infty$ with $a_{i}=a_{r_{i}}$ such that $m_{r_{i}}^{a_{i}}=0$ and $a_{i} \rightarrow a<\infty$. The same argument as before implies that $g_{i} \rightarrow g$, which is bounded on $e_{j}$ for all $3 \leq j \leq s$, and $g=\psi_{2}+h$ on $e_{2}$ for some bounded harmonic function $h$. Morever, if we set $\eta_{i}=g_{i}+a_{i} \psi_{1}$ on $e_{1} \cap B_{p}\left(r_{i}\right)$, then $\eta_{i}$ is harmonic with boundary conditions $\eta_{i}=0$ on $e_{1} \cap \partial B_{p}\left(r_{i}\right)$ and $\eta_{i}=g_{i}$ on $\partial e_{1}$. Hence, by taking the limit, $\lim _{i \rightarrow \infty} \eta_{i}=\eta=g+a \psi_{1}$ for some harmonic function $\eta$ which is bounded on $e_{1}$. This proves the 
existence of a harmonic function $g$ on $M$ which is bounded on $e_{j}$ for $3 \leq j \leq s$. Also $g-\psi_{2}$ is bounded on $e_{2}$, and $g+a \psi_{1}$ is bounded on $e_{1}$ for some $a>0$.

Clearly, for any end $e_{j}$ for $2 \leq j \leq s$ we can find a harmonic function $g_{j}$ such that $g_{j}-\psi_{1}$ is bounded on $e_{1}, g_{j}+a_{j} \psi_{j}$ is bounded on $e_{j}$ for some $a_{j}>0$, and $g_{j}$ is bounded on both sides on all the other ends. We now claim that the set $\left\{1, g_{2}, \cdots, g_{s}\right\}$ forms a linearly independent set. In fact, let us consider the identity

$$
b=\sum_{j=2}^{s} b_{j} g_{j} .
$$

Evaluating at the sequence of points $\left\{q_{k}^{i}\right\}$ and letting $k \rightarrow \infty$ we see that $b_{i} a_{i}=0$. Since $a_{i}>0$, this implies that $b_{i}=0$. Hence $b=0$. This establishes (2.1).

Remark 2.1. Let $\mathscr{H}^{\prime}(M)$ be the space spanned by constant functions and all the harmonic functions constructed in the proof of the theorem for the ends of $M \backslash B_{p}(R)$ for any $R>0$. Then $\mathscr{H}^{\prime}(M) \subset \mathscr{H}^{0}(M)$, and the number of ends of $M$ is less than or equal to the dimension of $\mathscr{H}^{\prime}(M)$.

Remark 2.2. Let us point out that for a fixed $R>0$, because of the behavior of the harmonic functions $h_{i}$ and $g_{j}$ constructed in the theorem, their linear combinations are also bounded on one side on each end.

\section{Applications to Riemannian geometry}

In this section we will consider the manifold $M^{n}$ whose Ricci curvature has a lower bound given by $-k(r)$, where $k(r)$ is a nonnegative nonincreasing continuous function of the distance $r$ to a fixed point which satisfies the integrability condition

$$
\int_{0}^{\infty} r^{n-1} k(r) d r<\infty .
$$

We will give an estimate on the dimension of the space $\mathscr{H}^{\prime}(M)$ in terms of $k$ and $n$, which implies an upper bound for the number of ends of $M$. For the definition of $\mathscr{H}^{\prime}(M)$, see Remark 2.1. Let us first derive some estimates concerning the Jacobi equation on such manifolds. The integrability conditions on $k$ for these estimates are weaker than that which is required for the upper bound of $\mathscr{H}^{\prime}(M)$. The following fact was proved by Abresch in [1]. However, an outline of the argument is included for the sake of completeness. 
Proposition 3.1. Let $k:[0, \infty) \rightarrow[0, \infty)$ be a continuous nonincreasing function such that $\int_{0}^{\infty} r k(r) d r<\infty$. For $R>0$, let $g$ be the solution of the equation

$$
g^{\prime \prime}(r)=k(R-r) g(r)
$$

on $[0, R]$ with initial conditions $g(0)=0$ and $g^{\prime}(0)=1$. Then there exists a constant $\beta>0$ depending only on $k$ such that

$$
\beta \frac{r}{R} \leq \frac{g(r)}{g(R)} \leq 1 .
$$

Proof. Define $k_{1}(r)=\int_{r}^{\infty} k(s) d s$. By the assumption on $k$ it is easy to see that $k_{1}$ is well defined and $\int_{0}^{\infty} k_{1}(r) d r=\int_{0}^{\infty} r k(r) d r$ is finite. Let $u_{R}$ be the solution of

$$
\phi^{\prime}=\phi^{2}-k(r)
$$

on $[0, R]$ with boundary condition $u_{R}(R)=0$. Since $\phi=0$ is a subsolution and $k_{1}$ is a supersolution, it can be shown that $0 \leq u_{R} \leq k_{1}$ on $[0, R]$. Therefore $u=\lim _{R \rightarrow \infty} u_{R}$ exists and satisfies $u^{\prime}=u^{2}-k$ on $[0, \infty)$, and $0 \leq u(r) \leq k_{1}(r)$ for all $r \geq 0$. In particular,

$$
\int_{0}^{\infty} u(r) d r \leq \int_{0}^{\infty} k_{1}(r)<\infty .
$$

If we define $z(r)=\exp \left(-\int_{0}^{r} u(s) d s\right)$, then one checks that $z(0)=1$, $z(r)>0$, and $z^{\prime \prime}=k z$. Now let $R>0$ be fixed and let $g$ be the solution of

$$
g^{\prime \prime}(r)=k(R-r) g(r)
$$

on $[0, R]$, with initial conditions $g(0)=0$ and $g^{\prime}(0)=1$. Note that $g$ is increasing and $g(r) / g(R) \leq 1$. To prove the lower bound, let us define $h(r)=g(R-r) / g(R)$. Then $h$ satisfies

$$
h^{\prime \prime}(r)=k(r) h(r)
$$

on $[0, R]$, with boundary conditions $h(0)=1$ and $h(R)=0$. By the maximum principle, $z \geq h$ on $[0, R]$, and hence $z-h$ is convex. Therefore if $r \in[0, R]$, we have

$$
z(r)-h(r) \leq \frac{r}{R}(z(R)-h(R))+\left(1-\frac{r}{R}\right)(z(0)-h(0)) \leq \frac{r}{R} z(R)
$$

and

$$
h(r) \geq z(r)-\frac{r}{R} z(R) \geq z(R)\left(1-\frac{r}{R}\right) \geq \beta\left(1-\frac{r}{R}\right),
$$

where $\beta=\exp \left(-\int_{0}^{\infty} u(s) d s\right)>0$. By the definition of $h$, we conclude that

$$
\frac{g(r)}{g(R)}=h(R-r) \geq \beta \frac{r}{R}
$$


for all $r \in[0, R]$. This completes the proof of the proposition.

Lemma 3.1. Let $M^{n}$ be a complete noncompact manifold. Suppose the Ricci curvature of $M$ satisfies

$$
\operatorname{Ric}_{M}(x) \geq-k(r(x))
$$

where $k:[0, \infty) \rightarrow[0, \infty)$ is a continuous nonincreasing function of the distance $r(x)=d(p, x)$ to a fixed point $p \in M$, such that $\int_{0}^{\infty} r k(r) d r<$ $\infty$. Given $0<\delta<1$, there exists a constant $C_{1}>0$ such that for all $R>0$ and $x \in \partial B_{p}(R)$,

$$
V_{x}(\delta R) \geq C_{1} R
$$

Proof. For $x \in \partial B_{p}(R)$ with $R>4$, let $\gamma$ be a minimal geodesic from $p$ to $x$, parametrized by arclength with $\gamma(x)=R$. Denote $q=\gamma(1)$. For $y \in B_{x}(R)$ and $d(x, y)=t$, the triangle inequality implies that $d(p, y) \geq R-t$. Hence $\operatorname{Ric}_{M}(y) \geq-k(R-t)$, because $k$ is nonincreasing. Let $g:[0, R] \rightarrow \mathbf{R}$ be the solution of

$$
g^{\prime \prime}(t)=\frac{k(R-t)}{n-1} g(t),
$$

with initial conditions $g(0)=0$ and $g^{\prime}(0)=1$. Using the same argument as in [6] and applying Proposition 3.1, we have

$$
\begin{aligned}
\frac{V_{q}(1)}{V_{x}(R-2)} & \leq \frac{V_{x}(R)-V_{x}(R-2)}{V_{x}(R-2)} \leq \frac{\int_{R-2}^{R} g^{n-1}(t) d t}{\int_{0}^{R-2} g^{n-1}(t) d t} \\
& =\frac{\int_{R-2}^{R}(g(t) / g(R))^{n-1} d t}{\int_{0}^{R-2}(g(t) / g(R))^{n-1} d t} \leq C_{2} R^{-1},
\end{aligned}
$$

where $C_{2}$ is a constant depending only on $n$ and $k$.

Hence

$$
V_{x}(R) \geq C_{2}^{-1}\left(\inf _{y \in \partial B_{p}(1)} V_{y}(1)\right) R .
$$

On the other hand, using a similar argument, we have

$$
\frac{V_{x}(R)}{V_{x}(\delta R)} \leq \frac{\int_{0}^{R} g^{n-1}(t) d t}{\int_{0}^{\delta R} g^{n-1}(t) d t} \leq C_{3},
$$

where $C_{3}$ is a constant depending only on $n, \delta$, and $k$. Combining this with (3.1), the result follows. q.e.d.

We will now give an estimate for the gradient of the barriers constructed in $\S 1$. Let us recall a localized version of Yau's gradient estimate for harmonic functions which was proved by Cheng-Yau [9] (see also [22]). 
Lemma 3.2. Let $M$ be an n-dimensional complete Riemannian manifold which may have boundary. Suppose $B_{x}(2 R)$ is a geodesic ball centered at $x \in M$ with radius $2 R$. Let us assume that $B_{x}(2 R) \cap \partial M=\varnothing$. If $f$ is a positive harmonic function defined on $B_{x}(2 R)$ and if the Ricci curvature of $M$ satisfies $\mathrm{Ric}_{M} \geq-k$ on $B_{x}(2 R)$ for some constant $k \geq 0$, then there exists a constant $C>0$ depending only on $n$ such that

$$
|\nabla f|^{2}(y) \leq C\left(R^{-2}+k\right) f^{2}(y)
$$

for all $y \in B_{x}(R)$.

Lemma 3.3. Let $M^{n}$ be a complete noncompact manifold and $p$ a fixed point. Suppose there is a constant $C_{4}>0$, such that the Ricci curvature of $M$ satisfies $\operatorname{Ric}_{M}(x) \geq-C_{4} r^{-2}(x)$, where $r(x)=d(p, x)$ is the distance function to $p$. Let $R_{0}>0$, and let $f$ be a harmonic function on $M \backslash B_{p}\left(R_{0}\right)$ such that $f=\lim _{i \rightarrow \infty} f_{i}$, where $f_{i}$ is the harmonic function on $B_{p}\left(R_{i}\right) \backslash B_{p}\left(R_{0}\right)$ satisfying $\left.f_{i}\right|_{\partial B_{p}\left(R_{0}\right)}=0,\left.f_{i}\right|_{\partial B\left(R_{i}\right)}=C_{i}>0$, with $R_{i} \rightarrow \infty$. Then there is a constant $C_{6}$ depending only on $n$ and $C_{4}$ such that for $R>R_{0}$

$$
\sup _{\partial B_{p}(R)}|\nabla f|^{2} \leq \frac{C_{6}}{\inf _{x \in \partial B_{p}(R)} V_{x}(R / 5)}\left(\sup _{\partial B_{p}(R)} f\right) \int_{\partial B_{p}\left(R_{0}\right)} \frac{\partial f}{\partial r} .
$$

Proof. For simplicity of notation, let us assume that $R_{0}=1$. By Lemma 1.4, we have

$$
\begin{aligned}
\int_{B_{p}(R)-B_{p}(1)}|\nabla f|^{2} & \leq 4\left(\sup _{B_{p}(R)-B_{p}(1)} f\right) \int_{\partial B_{p}(1)} \frac{\partial f}{\partial r} \\
& =4\left(\sup _{\partial B_{p}(R)} f\right) \int_{\partial B_{p}(1)} \frac{\partial f}{\partial r} .
\end{aligned}
$$

The Bochner formula implies that, for all $x \in \partial B_{p}(R)$ and $R>1$,

$$
\Delta|\nabla f| \geq-\alpha(R)|\nabla f|
$$

on $B_{x}(R / 5)$, where $-\alpha=-\alpha(R)$ is the lower bound of the Ricci curvature of $B_{p}(6 R / 5) \backslash B_{p}(4 R / 5)$. In particular, $\alpha \leq 2 C_{4} R^{-2}$. Since $u(y, t)=$ $\exp (-\alpha t)|\nabla f|(y)$ is a subsolution of the heat equation on $B_{x}(R / 5) \times$ $[0, \infty)$, we can apply the mean value inequality in [21] to $u(y, t)$ on $B_{x}(R / 5) \times\left[0, R^{2} / 100\right]$ and obtain

$$
|\nabla f|^{2}(x) \leq \frac{C_{5}}{V_{x}(5 / R)} \int_{B_{x}(5 / R)}|\nabla f|^{2},
$$


where $C_{5}$ is a constant depending only on $n$ and $C_{4}$. Hence, by (3.2), there exists a constant $C_{6}$ depending only on $n$ and $k$ such that

$$
\begin{aligned}
\sup _{\partial B_{p}(R)}|\nabla f|^{2} & \leq \frac{C_{5}}{\inf _{x \in \partial B_{p}(R)} V_{x}(R / 5)} \int_{B_{p}(6 R / 5)}|\nabla f|^{2} \\
& \leq \frac{C_{5}}{\inf _{x \in \partial B_{p}(R)} V_{x}(R / 5)}\left(\sup _{\partial B_{p}(6 R / 5)} f\right) \int_{\partial B_{p}(1)} \frac{\partial f}{\partial r} \\
& \leq \frac{C_{6}}{\inf _{x \in \partial B_{p}(R)} V_{x}(R / 5)}\left(\sup _{\partial B_{p}(R)} f\right) \int_{\partial B_{p}(1)} \frac{\partial f}{\partial r}
\end{aligned}
$$

where we have used Lemma 3.2 and the curvature assumption. q.e.d.

We will now apply this result to some particular cases.

Corollary 3.1. Let $M^{n}$ be a complete noncompact manifold satisfying the curvature assumption of Lemma 3.1, and let $f$ be a harmonic function as in Lemma 3.3. Then $f$ has bounded gradient. In particular, every harmonic function in $\mathscr{H}^{\prime}(M)$ has bounded gradient.

Proof. Since $\int_{0}^{\infty} r k(r) d r<\infty$ and $k$ is nonincreasing, $k$ must satisfy $k(r)=o\left(r^{-2}\right)$. Following the same notation as in the proof of Lemma 3.3, suppose $|\nabla f|$ is not bounded. Then there exists $R_{i} \rightarrow \infty$ such that

$$
\limsup _{R_{i} \rightarrow \infty} \sup _{B_{p}\left(R_{i}\right) \backslash B_{p}(1)}|\nabla f|=\infty \text {. }
$$

We may also assume that $\sup _{B_{p}\left(R_{i}\right) \backslash B_{p}(1)}|\nabla f|=\sup _{\partial B_{p}\left(R_{i}\right)}|\nabla f|$. By Lemmas 3.1 and 3.3, there is a constant $C_{7}$ independent of $i$ such that

$$
\begin{aligned}
\sup _{\partial B_{p}\left(R_{i}\right)}|\nabla f|^{2} & \leq C_{7} \sup _{B_{p}\left(R_{i}\right) \backslash B_{p}(1)}|\nabla f| \int_{\partial B_{p}(1)} \frac{\partial f}{\partial r} \\
& =C_{7} \sup _{\partial B_{p}\left(R_{i}\right)}|\nabla f| \int_{\partial B_{p}(1)} \frac{\partial f}{\partial r},
\end{aligned}
$$

which implies that $\sup _{\partial B_{p}\left(R_{i}\right)}|\nabla f|$ are uniformly bounded; this is a contradiction. q.e.d.

A similar argument will also prove the following corollary.

Corollary 3.2. Let $M$ and $f$ be as in Lemma 3.3. Suppose that $\lim _{x \rightarrow \infty} V_{x}(r(x) / 5)=\infty$. Then $|\nabla f|(x)=o(r(x))$.

Theorem 3.1. Let $M^{n}$ be a complete noncompact manifold and $p \in$ $M$ a fixed point such that $\operatorname{Ric}_{M}(x) \geq-k(r(x))$, where $r(x)=d(p, x)$. Suppose that $k:[0, \infty) \rightarrow[0, \infty)$ is a nonincreasing continuous function such that $\int_{0}^{\infty} r^{n-1} k(r) d r<\infty$. Then $\operatorname{dim} \mathscr{H}^{\prime}(M) \leq C(n, k)$ for some 
constant $C$ depending only on $n$ and $k$. In particular, the number of ends of $M$ is less than or equal to $C(n, k)$.

Proof. If $n=2$, the theorem follows from [1], hence we may assume that $n \geq 3$. Let $\bar{M}$ be the Ricci model of dimension $n$ (see [7]) which has Ricci curvature in the radial direction given by $-k(\bar{d}(\bar{p}, \bar{x}))$, where $\bar{p}$ is the pole of $\bar{M}$ and $\bar{d}$ is the distance function of $\bar{M}$. Let us denote $\bar{A}(r)$ to be the area of $\partial B_{\bar{p}}(r)$. By the assumption on $k$ we see that $\bar{A}(r) / r^{n-1}$ is bounded from above and below by some positive constants. In fact this is true if $\int_{0}^{\infty} r k(r) d r<\infty$ (see [11]). Hence the function

$$
\phi(r)=\int_{r}^{\infty} \frac{1}{\bar{A}(s)}\left(\int_{s}^{\infty} \bar{A}(t) k(t) d t\right) d s
$$

is well defined on $(0, \infty)$. Clearly, $\phi$ is positive and satisfies $\lim _{r \rightarrow \infty} \phi(r)$ $=0$ and $\phi^{\prime} \leq 0$. Let $g$ be the function on $M$ such that $g(x)=$ $\phi(r(x))$. Then

$$
\Delta g=\phi^{\prime} \Delta r+\phi^{\prime \prime} \geq \frac{\bar{A}^{\prime}}{\bar{A}} \phi^{\prime}+\phi^{\prime \prime}=k
$$

in the sense of distribution. This follows from the fact that $\phi^{\prime} \leq 0$ and that $\Delta r \leq \bar{A}^{\prime} \bar{A}$ in the sense of distribution. Let $R_{0}>0$ be such that $\phi\left(R_{0}\right) \leq \frac{1}{2}$, and note that $R_{0}$ depends only on $n$ and $k$. If $f$ is a nonconstant harmonic function in $\mathscr{H}^{\prime}(M)$, then $f$ has bounded gradient by Corollary 3.1. We now claim that

$$
\sup _{M}|\nabla f| \leq 2 \sup _{B_{p}\left(R_{0}\right)}|\nabla f| .
$$

Without loss of generality, we may assume that $\sup _{M}|\nabla f|=1$. Then

$$
\Delta(|\nabla f|+g) \geq-k|\nabla f|+k \geq 0 .
$$

By the definition of $\mathscr{C}^{\prime}(M)$, there exists $R_{1}>R_{0}$ such that $f=\sum_{i=1}^{m} v_{i}$, where each $v_{i}$ is bounded on one side of each end of $M \backslash B_{p}\left(R_{1}\right)$. Let $E$ be an end of $M \backslash B_{p}\left(R_{1}\right)$. If $v$ is a harmonic function defined on $M$ which is positive on $E$ and if $x$ is a point in $E$ with $r(p, x) \geq 2 R_{1}$, then by applying Lemma 3.2 to the ball $B_{x}(r(p, x) / 2)$ and using the curvature assumption there is a constant $C_{8}$ independent of $v$ such that

$$
|\nabla v|(x) \leq C_{8} r^{-1}(p, x) v(x) .
$$

Since all the $v_{i}$ 's are bounded on one side on $E$, there are constants $a_{1}, \cdots, a_{m}$ and $\varepsilon_{i}= \pm 1$ such that the harmonic functions $u_{i}=a_{i}+$ $\varepsilon_{i} v_{i}$ are positive on $E$. Hence, by applying (3.4) to $u_{1}, \cdots, u_{m}$ we can 
estimate the gradient of $f$ by

$$
|\nabla f| \leq \sum_{i=1}^{m}\left|\nabla v_{i}\right|=\sum_{i=1}^{m}\left|\nabla u_{i}\right|<C_{8} r^{-1}(p, x) \sum_{i=1}^{m} u_{i}(x) .
$$

For any given $\varepsilon>0$, inequality (3.3), the fact that $\lim _{x \rightarrow \infty} g(x)=0$, and the maximum principle imply

$$
|\nabla f|(x)+g(x)-\left(\sup _{\partial E}(|\nabla f|+g)\right) \leq C_{8} \varepsilon\left(\sum_{i=1}^{m} u_{i}(x)+1\right)
$$

for all $x \in E$. Letting $\varepsilon \rightarrow 0$, we conclude that

$$
\sup _{E}(|\nabla f|+g) \leq \sup _{\partial E}(|\nabla f|+g) .
$$

Since $E$ is any end of $M \backslash B_{p}\left(R_{1}\right)$, we have

$$
\sup _{M \backslash B_{p}\left(R_{1}\right)}(|\nabla f|+g) \leq \sup _{\partial B_{p}\left(R_{0}\right)}(|\nabla f|+g)
$$

We now claim that in fact

$$
\sup _{M \backslash B_{p}\left(R_{0}\right)}(|\nabla f|+g) \leq \sup _{\partial B_{p}\left(R_{1}\right)}(|\nabla f|+g) .
$$

Assuming the contrary, by the maximum principle and the fact the $|\nabla f|+g$ is subharmonic on $M \backslash B_{p}\left(R_{0}\right)$, $\sup _{M \backslash B_{p}\left(R_{0}\right)}(|\nabla f|+g)$ must be attained at infinity and so

$$
\sup _{M \backslash B_{p}\left(R_{1}\right)}(|\nabla f|+g)>\sup _{\partial B_{p}\left(R_{1}\right)}(|\nabla f|+g) .
$$

However, this violates (3.6). In particular, this implies that

$$
\begin{aligned}
\sup _{M \backslash B_{p}\left(R_{0}\right)}(|\nabla f|+g) & \leq \sup _{\partial B_{p}\left(R_{0}\right)}(|\nabla f|+g) \leq \sup _{\partial B_{p}\left(R_{0}\right)}|\nabla f|+\frac{1}{2} \\
& =\sup _{\partial B_{p}\left(R_{0}\right)}|\nabla f|+\frac{1}{2} \sup _{M}|\nabla f| .
\end{aligned}
$$

Therefore

$$
\sup _{M}|\nabla f| \leq 2 \sup _{B_{p}\left(R_{0}\right)}|\nabla f| .
$$

Let us now consider the codimension-1 subspace $\mathscr{H}_{p}^{\prime}(M)$ of $\mathscr{H}^{\prime}(M)$ defined by

$$
\mathscr{H}_{p}^{\prime}(M)=\left\{f \in \mathscr{H}^{\prime}(M) \mid f(p)=0\right\} .
$$

For any $f \in \mathscr{H}_{p}^{\prime}(M)$, the fundamental theorem of calculus gives

$$
\sup _{B_{p}\left(4 R_{0}\right)} f^{2} \leq 16 R_{0}^{2} \sup _{B_{p}\left(4 R_{0}\right)}|\nabla f|^{2}
$$


which together with (3.7), implies

$$
\sup _{B_{p}\left(4 R_{0}\right)} f^{2} \leq 64 R_{0}^{2} \sup _{B_{p}\left(R_{0}\right)}|\nabla f|^{2} .
$$

Applying Lemma 3.2 to the function $f+\sup _{B_{p}\left(2 R_{0}\right)}|f|$ we conclude that

$$
\begin{aligned}
\sup _{B_{p}\left(4 R_{0}\right)} f^{2} & \leq 32 R_{0}^{2} \sup _{B_{p}\left(r_{0}\right)}|\nabla f|^{2} \\
& \leq C_{9}\left(1+R_{0}^{2} k(0)\right) \sup _{B_{p}\left(R_{0}\right)}\left(f+\sup _{B_{p}\left(2 R_{0}\right)}|f|\right)^{2} \\
& \leq 4 C_{9}\left(1+R_{0}^{2} k(0)\right) \sup _{B_{p}\left(2 R_{0}\right)} f^{2}
\end{aligned}
$$

where $C_{9}$ is a constant depending only on $n$. However, the mean value inequality of Li-Schoen [17] when applied to the nonnegative subharmonic function $|f|$ asserts that there exists a constant $C_{10}(n)>0$ depending only on $n$ such that

$$
V_{p}\left(4 R_{0}\right) \sup _{B_{p}\left(2 R_{0}\right)} f^{2} \leq \exp \left(C_{10}(n)\left(1+R_{0} \sqrt{k(0)}\right)\right) \int_{B_{p}\left(4 R_{0}\right)} f^{2},
$$

where $V_{p}\left(4 R_{0}\right)$ denotes the volume of $B_{p}\left(4 R_{0}\right)$. Hence, combining with (3.8), we have

$$
V_{p}\left(4 R_{0}\right) \sup _{B_{p}\left(4 R_{0}\right)} f^{2} \leq C_{11} \exp \left(C_{12} R_{0} \sqrt{k(0)}\right) \int_{B_{p}\left(4 R_{0}\right)} f^{2},
$$

with $C_{11}, C_{12}$ depending only on $n$. On the other hand, a lemma of the first author [15, Lemma] implies that there exists a function $f_{0}$ in $\mathscr{H}_{p}^{\prime}(M)$ such that

$$
\operatorname{dim} \mathscr{H}_{p}^{\prime}(M) \int_{B_{p}\left(4 R_{0}\right)} f_{0}^{2} \leq v_{p}\left(4 R_{0}\right) \sup _{B_{p}\left(4 R_{0}\right)} f_{0}^{2} .
$$

Hence applying (3.9) to $f_{0}$ yields the estimate

$$
\operatorname{dim} \mathscr{H}_{p}^{\prime}(M) \leq C_{11} \exp \left(C_{12} R_{0} \sqrt{k(0)}\right)
$$

Therefore,

$$
\operatorname{dim} \mathscr{H}^{\prime}(M) \leq C_{11} \exp \left(C_{12} R_{0} \sqrt{k(0)}\right)+1,
$$

as to be proven. The estimate on the number of ends follows from Theorem 2.1. q.e.d. 
When the Ricci curvature is nonnegative outside a compact set, say $B_{p}(1)$, then one can actually estimate the dimension of a larger space, $\mathscr{H}^{0}(M)$, by a much simpler argument. In that case, we can take $g$ to be 0 and $R_{0}=1$ in the proof of Theorem 3.1 and we do not need to use Lemma 3.3. Let us just state the theorem without proof.

Theorem 3.2. Let $M$ be an $n$-dimensional complete noncompact manifold which has nonnegative Ricci curvature outside a unit geodesic ball $B_{p}(1)$ centered at $p \in M$. If $k \geq 0$ is a constant such that the Ricci curvature on $B_{p}(1)$ is bounded from below by $-k$, then there exist constants $C_{13}, C_{14}>0$ depending only on $n$, such that

$$
\operatorname{dim} \mathscr{H}^{0}(M) \leq C_{13} \exp \left(C_{14} \sqrt{k}\right)+1 .
$$

In particular, the number of ends of $M$ is not greater than $C_{13} \exp \left(C_{14}(\sqrt{k})\right.$ +1 .

We would like to remark that when $M$ has nonnegative Ricci curvature everywhere, we may take the ball $B_{p}(1)=\{p\}$ in (3.7) and obtain the estimate

$$
\sup _{M}|\nabla f| \leq|\nabla f|(p) .
$$

However, since $|\nabla f|$ is a subharmonic function on $M$, the maximum principle implies that $|\nabla f|$ must be identically constant. Applying the Bochner formula to the constant function $|\nabla f|$ again, one concludes that $\nabla f$ is a parallel vector field on $M$. This recovers the consequence of the splitting theorem of Cheeger-Gromoll asserting that if $M$ has more than one end, then it must be the product of a compact manifold and the real line.

\section{Applications to Kähler geometry}

Throughout this section, we will consider complete Kähler manifolds which are noncompact without boundary. We will apply Theorem 2.1 to study function-theoretic properties of the ends.

Theorem 4.1. Let $M$ be a Kähler manifold of complex dimension $m$. Suppose $R(x)$ is a pointwise lower bound of the Ricci curvature of $M$, i.e., $\operatorname{Ric}_{i j}(x) \geq R(x) g_{i j}$ for $x \in M$. Let $R_{-}(x)=\max \{-R(x), 0\}$ denote the negative part of $R$. If $\int_{M} R_{-} d V<\infty$, and the $L^{q}$-norm of $R_{-}$over the geodesic ball of radius $r$ centered at some fixed point $p \in M$ satisfies

$$
\int_{B_{p}(r)} R_{-}^{q} d V=o\left(r^{\beta(q-1)}\right)
$$


for some $q>m$ and $\beta<2 /(m-2)$, then $M$ has at most one nonparabolic end.

Proof. If $M$ has more than one nonparabolic end, then by Theorem 2.1 there exists a nonconstant bounded harmonic function $f$ on $M$ with finite Dirichlet integral. However, by a lemma of the first author [16, Lemma 3.1], the function $f$ must be pluriharmonic. On the other hand, it was shown by Li-Yau [23] (also see [16]) that under the curvature hypotheses there are no nonconstant positive pluriharmonic functions. This contradicts the assumption that $M$ has more than one nonparabolic end.

Theorem 4.2. There exists a constant $\delta(m)>0$ depending only on $m$ such that if $M$ is a nonparabolic Kähler manifold of complex dimension $m$ with Ricci curvature satisfying $\operatorname{Ric}_{M}(x) \geq-\delta(r(x))^{-2}$, where $r(x)=$ $d(p, x)$ is the distance function to a fixed point $p \in M$, then $M$ has only one end.

Proof. Let us assume that $M \backslash B_{p}$ (1) has more than one end. The nonparabolicity and Theorem 2.1 imply that there exists a nonconstant positive harmonic function $f$ by using the construction in $\S 2$. It is clear that if we can establish that the Dirichlet integral of $f$ satisfies

$$
\int_{B_{p}(r) \cap E}|\nabla f|^{2}=o\left(r^{2}\right)
$$

for each end $E$, then the argument in the proof of Theorem 4.1 applies to this case. However, Corollary 3.2, the proof of Theorem 2.5 in [17], and the construction of $f$ imply that $f(x)=o\left((r(x))^{2}\right)$. Property (4.1) now follows from Lemmas 1.3 and 1.4.

\section{References}

[1] U. Abresch, Lower curvature bounds, Toponogov's theorem and bounded topology. I, Ann. Sci. École Norm. Sup. (4) 19 (1985) 651-670.

[2] __ Lower curvature bounds, Toponogov's theorem, and bounded topology. II, Ann. Sci. École Norm. Sup. (4) 20 (1987) 475-502.

[3] M. Cai, Ends of Riemannian manifolds with nonnegative Ricci curvature outside a compact set, preprint.

[4] J. Cheeger \& D. Gromoll, The splitting theorem for manifolds of nonnegative Ricci curvature, J. Differential Geometry 6 (1971) 119-128.

[5] _ On the structure of complete manifolds of nonnegative curvature, Ann. of Math. (2) 92 (1972) 413-443.

[6] J. Cheeger, D. Gromoll \& M. Taylor, Finite propagation speed, kernel estimates for functions of the Laplace operator, and the geometry of complete Riemannian manifolds, J. Differential Geometry 17 (1983) 15-53.

[7] J. Cheeger \& S. T. Yau, A lower bound for the heat kernel, Comm. Pure Appl. Math. 34 (1981) 465-480.

[8] S. Y. Cheng, Finite dimensionality of the spaces of positive and bounded harmonic functions, preprint. 
[9] S. Y. Cheng \& S. T. Yau, Differential equations on Riemannian manifolds and their geometric applications, Comm. Pure Appl. Math. 28 (1975) 333-354.

[10] H. Donnelly, Bounded harmonic functions and positive Ricci curvature, Math. Z. 191 (1986) 559-565.

[11] R. Green \& H. Wu, Function theory on manifolds which possess a pole, Lecture Notes in Math., Vol. 699, Springer, Berlin, 1979.

[12] A. A. Grigor' yan, $A$ criterion for the existence on a Riemannian manifold of a nontrivial bounded harmonic function with finite Dirichlet integral, Soviet Math. Dokl. 35 (1987) 339-341.

[13] A. Kasue, $A$ compactification of a manifold with asymptotically nonnegative curvature, Ann. Sci. École Norm. Sup. (4) 21 (1988) 593-622.

[14] _ Harmonic functions with growth conditions on a manifold of asymptotically nonnegative curvature. I, preprint.

[15] P. Li, On the Sobolev constant and the p-spectrum of a compact Riemannian manifold, Ann. Sci. École Norm. Sup. (4) 13 (1980) 451-469.

[16] _ On the structure of complete Kähler manifolds with nonnegative curvature near infinity, Invent. Math. 99 (1990) 579-600.

[17] P. Li \& R. Schoen, $L^{p}$ and mean value properties of subharmonic functions on Riemannian manifolds, Acta Math. 153 (1984) 279-301.

[18] P. Li \& L. F. Tam, Positive harmonic functions on complete manifolds with non-negative curvature outside a compact set, Ann. of Math. (2) 125 (1987) 171-207.

[19] __ Symmetric Green's functions on complete manifolds, Amer. J. Math. 109 (1987) 1129-1154.

[20] _ Complete surfaces with finite total curvature, J. Differential Geometry 33 (1991) 139-168.

[21] _ , The heat equation and harmonic maps of complete manifolds, preprint.

[22] P. Li \& and S. T. Yau, On the parabolic kernel of the Schrödinger operator, Acta Math. 156 (1986) 153-201.

[23] _ Curvature and holomorphic mappings of complete Kähler manifolds, Compositio Math. 73 (1990) 125-144.

[24] Z.-D. Liu, Ball covering on manifolds with nonnegative Ricci curvature near infinity, preprint.

[25] T. J. Lyons, Instability of the Liouville property for quasi-isometric Riemannian manifolds and reversible Markov chains, J. Differential Geometry 26 (1987) 33-66.

[26] B. Malgrange, Existence et approximation des solutions der équations aux dérivées partielles et des équations de convolution, Ann. Inst. Fourier (Grenoble) 6 (1955) 271355.

[27] J. P. Sha \& D. G. Yang, Examples of manifolds of positive Ricci curvature, J. Differential Geometry 29 (1989) 95-103.

[28] _ Positive Ricci curvature on the connected sums of $\mathbf{S}^{n} \times \mathbf{S}^{m}$, preprint.

[29] N. Varopoulos, The Poisson kernel on positive curved manifolds, J. Funct. Anal. 44 (1981) 359-380.

[30] _ _ Potential theory and diffusion on Riemannian manifolds, Conference on Harmonic Analysis in Honor of Antoni Zygmund, Vols. I, II, Wadsworth Math. Ser., Wadsworth, Belmont, CA, 1983, 821-837.

[31] S. T. Yau, Harmonic functions on complete Riemannian manifolds, Comm. Pure Appl. Math. 28 (1975) 201-228. 
